\title{
Very Fast Current Diagnostic for Linear Pulsed Beams
}

\author{
Vincenzo Nassisi ${ }^{*}$, Domenico Delle Side and Vito Turco \\ Dipartimento di Matematica e Fisica, Lab. LEAS - Università del Salento and I.N.F.N. sect. of Lecce, Italy
}

\begin{abstract}
Fast current pulses manage lasers and particle accelerators and require sophisticate systems to be detected. At today Rogowski coils are well known. They are designed and built with a toroidal structure. In recently application, flat transmission lines are imploded and for this reason we develop a linear Rogowski coil to detect current pulses inside flat conductors. To get deep information from the system, it was approached by means of the theory of the transmission lines. The coil we build presents a resistance but it doesn't influence the rise time of the response, instead the integrating time. We also studied the influence of the magnetic properties of coil support. The new device was able to record pulses of more hundred nanoseconds depending on the inductance, load impedance and resistance of the coil. Furthermore, its response was characterized by a sub-nanosecond rise time ( $\sim 100 \mathrm{ps})$, The attenuation coefficient depends mainly on the turn number of the coil, while the quality of the response depends both on the manufacture quality of the coil and on the magnetic core characteristics. In biophysical applications often, a double line is employed in order to have a sample as control and a sample stressed by a light source. So, in this case we build two equal plane lines by $100 \Omega$ characteristic resistance connected in parallel. We diagnosed the current present in a line. The attenuation factor resulted to be $11,5 \mathrm{~A} / \mathrm{V}$.
\end{abstract}

\section{Introduction}

Rogowski coil is a well-known electric current detector since about a century[1]. Nevertheless, today its capabilities are still of great interest. TEA lasers and accelerator system need to detect fast current[2]. Also flat transmission lines utilised for radiofrequency biophysical experiments[3] need to control the current.

It was developed to detect variable currents fluxing in a conductor, which is responsible of the magnetic field around the conductor proper axis. In principle, the voltage provided by the coil is proportional to the input pulse current derivative and in order to obtain a sound measure of the originating signal, an integrating circuit is applied[4]. For high frequency signals, its response becomes self-integrating, providing an attenuation factor independent of the time duration or signal frequency. Moreover, the behaviour of the system is more complex for fast pulses due to the creating a capacitance with the metallic shield.

In the past, this detail has been analysed and developed by a few authors [5-7]. Under a few restrictions, the proposed device allows to justify its behaviour for fast pulses, large dimensions of the detector and manufacture quality.

To study our detector, we build two equal plane lines by $100 \Omega$ characteristic resistance connected in parallel. We diagnosed the current present in a line.

\footnotetext{
Corresponding author: vincenzo.nassisi@unisalento.it
}

\section{Theory}

The theory of the Rogowski coil is based on the main laws of electromagnetism.

\subsection{Rogowski Coil}

A current fluxing in a conductor generates a magnetic field dependent on distance $r$ from it and a toroidal inductor is influenced by the magnetic flux derivative. Fig. 1 shows a sketch of the system.

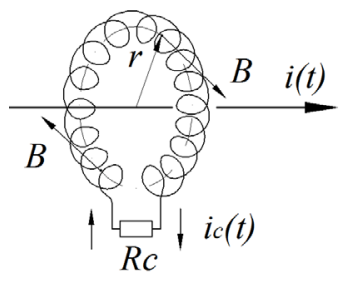

Fig. 1: Electrical circuit of a toroidal Rogowski coil with the input current in the centre.

Applying the Kirchhoff's equation [1, 8, 9], in the Laplace domain we have,

$$
p I_{i}(p) \frac{L_{c}}{n}=p L_{c} I(p)+R_{c} I(p)
$$

with $I_{i}(p)$ and $I(p)$ are the Laplace transforms of the input current $i(t)$ and of the coil current $i_{c}(t)$, respectivevely,e 
$L_{c}=\mu_{o} n^{2} A / 2 \pi r$ is the inductance of the coil, $n$ is the number of loops and $p$ is the Laplace variable. Now, hypothesizing a Heaviside function as current signal, i.e. $i(t)=I_{o} u(t)$, its transform is $I_{i}(p)=I_{o} / p$. Then the real output current results

$$
i_{c}(t)=\frac{I_{o}}{n} e^{-\frac{t}{\tau}} \cdot u(t)
$$

where $\tau=L_{c} / R_{c}$. This shows that the overall attenuation obtained in the detector with respect to the input current strongly depends on time due to the exponential factor. If we consider an observation time $T \ll \tau$ (more in general, $T$ is the pulse length of the input pulse), then the response on resistive load becomes

$$
i_{c}(t)=\frac{I_{o}}{n} \cdot u(t)
$$

The above result implies that the detector is also able to detect high frequency currents. Indeed, the steady-state response can be obtained from Eq. 1 substituting $p$ with $j \omega$, where $j$ is the imaginary unit and $\omega$ the frequency of the input current. The attenuation factor is

$$
\frac{i_{c}(t)}{i(t)}=\frac{1}{n} \frac{j \omega}{j \omega+\frac{R_{C}}{L_{C}}}
$$

then, to get a frequency independent response, in the steady-state case we observe that the attenuation is constant if $R_{c} / L_{c} \ll \omega$.

We stress that the results obtained do not depend on the shape of the coil loops neither on the area of each turn. Indeed, such assumptions are not satisfactory for short pulses.

The Rogowski coil we developed is devote for planar currents. This model is very useful, apart for laser discharges, also plane transmission lines. This last allows us to perform experiments of radiofrequency biophysical stresses [7, 9-11]. A sketch of a planar current is shown in Fig. 2. A current $i(t)$ in the conductor (width $a$ ) determines a current density that we call $j(t)=i(t) / a$.

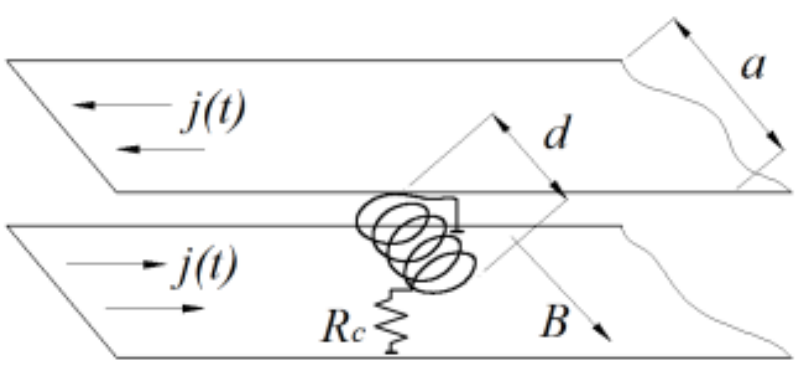

Fig. 2: Sketch of a Rogowski coil employed in a planar conductor.
Again, applying the Kirchhoff's equation, in the Laplace domain we have

$$
p I_{i}(p) \frac{L_{c}}{2 n} \frac{d}{a}=p L_{c} I(p)+R_{c} I(p)
$$

where $d$ is coil length and again $I_{i}(p)$ is the Laplace transform of the input current $i(t)$.

For an Heaviside-like input current, the corresponding Laplace transform is $I_{i}(p)=I_{o} / p$, and the final form of Eq. 5 becomes

$$
I_{o} \frac{L_{c}}{2 n} \frac{d}{a}=p L_{c} I(p)+R_{c} I(p)
$$

whose real solution is

$$
i_{c}(t)=\frac{1}{n} \frac{d}{a} I_{o} e^{-\frac{R_{C}}{L_{C}} t}
$$

and for $t \ll L_{c} / R_{c}$, the response will be time independent:

$$
i_{c}(t)=\frac{1}{n} \frac{d}{a} I_{o}
$$

This result is comparable to that obtained for the toroidal Rogowski coil described by Eq. 3, apart from the correction factor $d / 2 a$. Again, utilizing $j \omega$ instead of $p$ it is possible to get the steady-state response.

\subsection{Rogowski coil as distributed elements}

For fast pulses the above circuits are insufficient because they are unsuitable for transmit fast signals. So we consider the Rogowski coil as composed by distributed elements. It allows us to justify the presence of anomalies on the signal response generally due to imperfect manufacture.

To avoid interferences of stray signals, the coil is enclosed in a shielding box. It is a cracked metallic box operated on the ground conductor. The crack is realized by operating a slim slit on the conductor. The slit changes the current direction which wraps the coil, inducting again the same electromotive force, see Fig. 3.

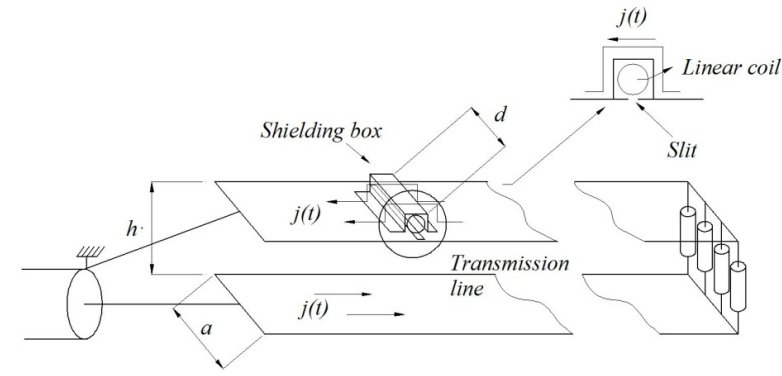

Fig. 3: Sketch of a flat transmission line with a linear Rogowski coil closed in the shielding box.

Now, the electromotive force can be considered distributed along the line and it can depend, apart from $t$, even from position, $x$ (with $x$ directed along the coil axis). 
So, to study it, let us to call $f(t, x)$ the electromotive force per unit length. Now, the presence of the metallic box exhibits a distributed capacitance along the coil. Let us to call $C(x)$ the capacitance per unit length. Even the inductance of the coil is distributed along the device and per unit length, it is $L(x)$, as well as that of the ground conductor, namely $L_{g}(x)$. Therefore, the device we analyse can be considered exactly as a transmission line, Fig 4.

Let us consider the electric circuit of this line by means of an infinite number of cells. A typical cell is illustrated in Fig. 4 where $f(t, x), L(x)$ and $C(x)$ are the voltage, the inductance and the capacitance per unit length, respectively. Besides, $L_{g}(x), R(x)$ and $G(x)$ are the inductance of the box conductors, the resistance of the coil wire and the conductance of the insulator per unit length, respectively.

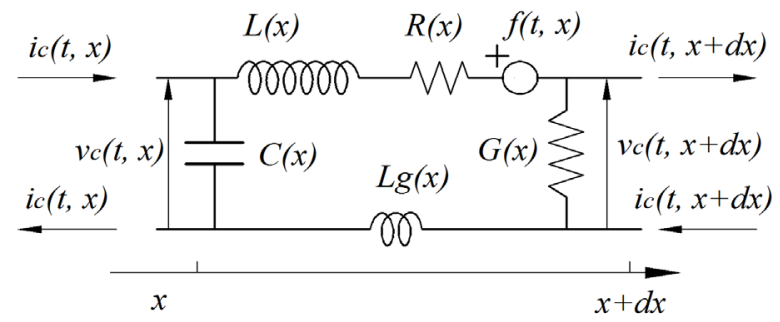

Fig. 4: Electric sketch of a Rogowski coil closed into the metallic box.

Then, applying Kirchhoff's equations, the differential of voltage and current for every cell to differential of position depend through the following equations:

$$
\begin{aligned}
& \frac{\partial v_{c}(t, x)}{\partial x}=-\{Z(t, x)\} i_{c}(t, x)-f(t, x) \\
& \frac{\partial i_{c}(t, x)}{\partial x}=-\{Y(t, x)\} v_{c}(t, x)
\end{aligned}
$$

with $\{Z(t, x)\}$ and $\{Y(t, x)\}$ the impedance and admittance operators of the circuit per unit length. They correspond to $\{Z\}=\left\{R+L \frac{d}{d t}+L_{g} \frac{d}{d t}\right\}$ and $\{Y\}=\{G+$ $\left.C \int d t\right\}$. Next, to simplify the solution, the resistance $R$, the inductance $L_{g}$ and the conductance $G$ are neglected and the line is represented by the circuit of Fig. 5. Operating the Laplace transform of Eq. 9, the impedance operator becomes $Z(p)=p L(x)$, the admittance one becomes $Y(p)=p C(x)$ and the electromotive force one becomes $F(p, x)=p I_{i}(p) L d / n a$.

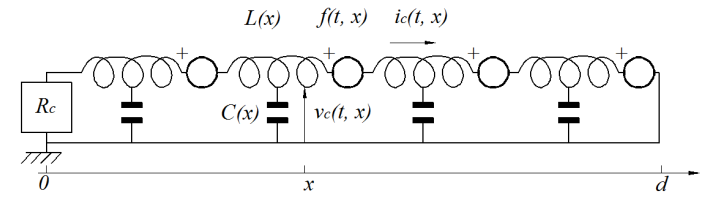

Fig. 5: Electric circuit of the Rogowski coil closed inside the metallic box. The voltage and the current along the coil are $v_{c}(t, x)$ and $i_{c}(t, x)$, respectively.
Theoretically, the last three parameters should be $x$ independent and this depends on the coil manufacture quality. Therefore, under the above constraints, the Laplace transform of Eq. 9 is:

$$
\begin{gathered}
V_{c}(x, p)=\frac{F(p) R_{C} R_{0}}{p L\left(R_{C}+R_{0}\right)} \cdot \frac{e^{-\tau_{0} p x}-e^{-\tau_{0} p(2 d-x)}}{1+\vartheta e^{-2 \tau_{0} p d}} \\
I_{C}(x, p)=\frac{F(p) R_{C}}{p L\left(R_{C}+R_{0}\right)} \frac{e^{-\tau_{0} p x}+e^{-\tau_{0} p(2 d-x)}}{1+\vartheta e^{-2 \tau_{0} p d}}-\frac{F(p)}{p L}
\end{gathered}
$$

where we have considered the line short-circuited at $x=$ $d$ and closed on resistive load $R_{c}$ at $x=0$. The parameter $\vartheta$ represents the reflection coefficient, namely $\left(R_{c}-R_{o}\right) /\left(R_{c}+R_{o}\right)$, where $R_{o}$ is the characteristic resistance of the line whose expression is $R_{o}=$ $\sqrt{Z(p) / Y(p)}$, while the propagation function per unit length is $-\tau_{0}$. In our conditions, the latter is a constant, $\tau_{0}=\sqrt{Z(p) Y(p)}=\sqrt{L C}$ and it expresses the delay per unit length. Because it is known to be of the order of $10^{-9}$ $s / m$ let us suppose that $\left|\vartheta e^{-2 \tau_{0} p d}\right|<1$ and we can operate the expansion

$$
\begin{gathered}
\left|\vartheta e^{-2 \tau_{0} p d}\right| \cong 1- \\
\vartheta e^{-2 \tau_{0} p d}+\vartheta^{2} e^{-4 \tau_{0} p d}-\vartheta^{3} e^{-6 \tau_{0} p d}+\cdots
\end{gathered}
$$

Substituting the Eq. 11 in the first Eq. 10, the voltage across $R_{c}$ is obtained placing $x=0$

$$
\begin{gathered}
V_{c}(x, p)=\frac{F(p) R_{C} R_{0}}{p L\left(R_{C}+R_{0}\right)} \quad\left[1-\vartheta_{V} e^{-2 \tau_{0} p d}+\right. \\
\left.\vartheta \vartheta_{V} e^{-4 \tau_{0} p d}-\vartheta^{2} \vartheta_{V} e^{-6 \tau_{0} p d} \ldots\right]
\end{gathered}
$$

with $\quad \vartheta_{V}=(1+\vartheta)=2 R_{c} /\left(R_{c}+R_{0}\right) . \quad$ Instead, substituting the Eq. 11 in the second Eq. 10, the current in $R_{c}$ is obtained again placing $x=0$

$$
\begin{gathered}
I_{c}(0, p)=\frac{F(p) R_{C}}{p L\left(R_{C}+R_{0}\right)}\left[1+\vartheta_{I} e^{-2 \tau_{0} p d}-\right. \\
\left.\vartheta \vartheta_{I} e^{-4 \tau_{0} p d}+\vartheta^{2} \vartheta_{I} e^{-6 \tau_{0} p d} \ldots\right]-\frac{F(p)}{p L}
\end{gathered}
$$

with $\vartheta_{I}=(1-\vartheta)=2 R_{0} /\left(R_{c}+R_{0}\right)$.

For an Heaviside-like input current, the corresponding electromotive force per unit length is constant

$$
F(p)=\frac{I_{0} L}{n} \frac{d}{a}
$$

Using Eq. 14, for $R_{c}=R_{0}$, we have $\vartheta=0$, and consequently $\vartheta_{V}=1$ as well as $\vartheta_{I}=1$. The response at $x=0$ becomes:

$$
\begin{gathered}
V_{c}(0, p)=\frac{I_{0} R_{0}}{2 p n} \frac{d}{a} \cdot\left[1-e^{-2 \tau_{0} p d}\right] \\
I_{c}(0, p)=\frac{I_{0}}{2 p n} \frac{d}{a} \cdot\left[1+e^{-2 \tau_{0} p d}\right]-\frac{I_{0}}{p n} \frac{d}{a}
\end{gathered}
$$




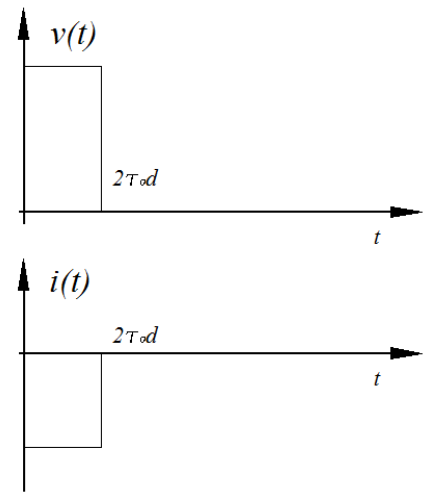

Fig. 6: Pictorial representation of the response waveforms for voltage and current with $x=0$ and $R_{c}=R_{0}$.

In the time domain, the result is a voltage and current pulse $2 \tau_{0} d$ long that corresponds to the time of a signal to propagate and came back for the whole line, Fig. 6. Nevertheless, this response does not match the input signal we want to detect. Instead, closing the Rogowski coil on a load resistor of low value such that $R_{c}<R_{0}$, it results that $-1<\vartheta<0,0<\vartheta_{V}<1$ and $1<\vartheta_{I}<2$. At $x=0$ the response is a voltage signal that decreases every $2 \tau_{0} d$ seconds of a little quantity, while the current signal is initially negative with the overlapping of a small positive signal every $2 \tau_{0} d$ seconds. Fig.7 shows a pictorial representation of the situation.

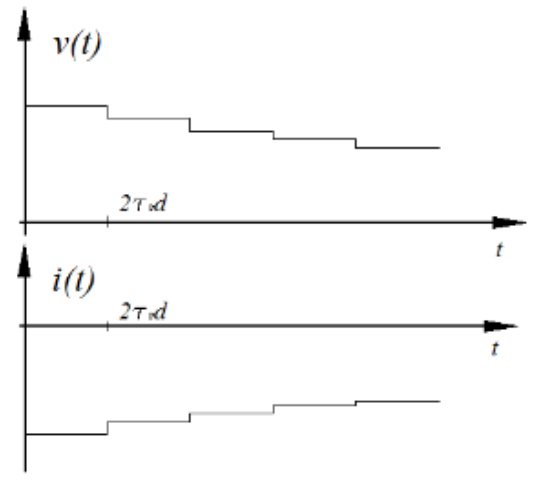

Fig. 10: Pictorial representation of the waveforms for voltage and current with $x=0$ and $R_{c}<R_{0}$.

This result is very interesting particularly if $R_{c} \ll R_{0}$ because we can have: $\vartheta \cong-1, \vartheta_{V} \cong 0$ and $\vartheta_{I} \cong 2$ and by the Eqs. 12 and 13 the response is approximately:

$$
\begin{aligned}
& V_{c}(0, p) \cong \frac{I_{0} R_{c}}{p n} \frac{d}{a} \\
& I_{c}(0, p) \cong-\frac{I_{0}}{p n} \frac{d}{a}
\end{aligned}
$$

Performing the inverse transformation in order to get the time dependent relations, the results are:

$$
\begin{aligned}
v_{R c}(t) & \cong \frac{I_{0} R_{C}}{n} \frac{d}{a} u(t) \\
i_{R c}(t) & \cong \frac{I_{0}}{n} \frac{d}{a} u(t)
\end{aligned}
$$

The relations obtained show that the theory developed give results very similar to the one expressed by Eq. 8 but now they deliver more information. Indeed, if the coil is not uniformly shaped, a different electromotive force will be induced at different positions and different values of inductance and capacitance are exhibited. In this case the propagation function per unit length isn't constant and an overlapped signal propagate along the line generating ripples on $R_{c}$. To attenuate the ripple, authors have suggested to apply some resistors along the coil in order to damp these undesirable signals [13]. In this case, the behaviour of the system improves but the value of the integrating time, that theoretically is $L d / R_{c}$, decreases. However, the insertion of the resistors must be realized without creating new loops and/or modify the capacitance values because they could introduce new elements that should modify the behaviour.

\subsection{Influence of the coil resistance}

During the development of the previous theory, the resistance of the coil wire has been neglected. The response of the Rogowski coil for a Heaviside-like signal is principally characterised by an attenuation coefficient and by a damping time. Then, considering a no-null value for $R$, the voltage on the load $R_{c}$ becomes

$$
\begin{gathered}
V_{c}(x, p)=\frac{F(p) R_{c}}{(p L+R)\left(1+\frac{R_{c}}{p L+R} p \tau_{0} \sqrt{1+\frac{R}{L p}}\right)} \\
{\left[\frac{e^{-\tau p x}}{1-\frac{Z-R_{C} \tau p}{Z+R_{C} \tau p} e^{-2 \tau p d}}-\frac{e^{+\tau p x} e^{-2 \tau p d}}{1-\frac{Z-R_{C} \tau p}{Z+R_{C} \tau p} e^{-2 \tau p d}}\right]}
\end{gathered}
$$

where the parameter $\tau=\tau_{0} \sqrt{1+R / p L}$. Then, before operating the inverse transformation, it is interesting to note that the part of Eq. 18 in square brackets governs the propagation of the signal. If we consider again the load resistance $R_{c} \sim 0$, condition necessary to get easily the result we expect, at $x=0$ the value of this part is 1 . Instead, the first part governs the attenuation factor. The inverse transformation of only this first part is very difficult, but the solution can be reached imposing that the coil resistance is generally small. Then, the expansion of the square root, considering only the first two terms is $\sqrt{1+\frac{R}{L p}} \cong 1+R / 2 L p$ and Eq. 18 then reads

$$
V_{c}(0, p) \cong \frac{F(p) R_{C}}{(p L+R)\left[1+\frac{R_{C}}{p L+R} p \tau_{0}\left(1+\frac{R}{2 L p}\right)\right]}
$$

Always for a Heaviside current signal as input, we have

$$
V_{c}(0, p) \cong \frac{L I_{0} R_{C}}{n(p L+R)\left[1+\frac{R_{C}}{p L+R} p \tau_{0}\left(1+\frac{R}{2 L p}\right)\right]} \frac{d}{a}
$$

The expression for $V_{c}(0, p)$ has got a pole that for $R_{c} \sim 0$ it amounts to $-R / L$. Therefore, performing the inverse 
transformation, the real voltage on $R_{c}$ exhibits an exponential behaviour, namely:

$$
v_{R c}(t)=\frac{I_{0} L R_{C}}{n\left(L+R_{C} \tau_{0}\right)} e^{-\frac{R t\left(2 L+R_{C} \tau_{0}\right)}{2 L\left(L+R_{C} \tau_{0}\right)}} \frac{d}{a} \cdot u(t)
$$

which for $R_{c} \sim 0$ becomes

$$
v_{R c}(t) \cong \frac{I_{0} R_{C}}{n} \frac{d}{a} e^{-\frac{R}{L} t} \cdot u(t)
$$

Comparing the Eq. 21 to Eqs. 17, we observe that a nonnegligible $R$ does not affect the attenuation coefficient but changes only the time behaviour. Therefore, during the design of a Rogowski coil, for high-precision measurements, it is necessary to consider both the values of $R$ and $R_{c}$ in order to find the current, while the voltage is still simply the current multiplied by only $R_{c}$.

\subsection{Influence of core characteristics}

Utilizing magnetic materials for the core of the coil its inductance changes as well as the electromotive force. An inductor with a core having only the real component of permeability, $\mu_{r}$, larger than 1 , has a $\mu_{r}$ times increased inductance value, but also the electromotive force increases of the same factor $\mu_{r}$. This behaviour could be considered as an advantage, since it reduces the effect of construction anomalies. In fact, loops of different cross section but with the same magnetic properties by constant diameter exhibit very near inductance values. Nevertheless, the use of a magnetic core involves a constant value of the permeability coefficient and of magnetic flux. In this way, a linear response is ensured.

In principle, the time response is not influenced by the permeability $\mu_{r}$, provided that the latter is constant. Instead, if the permeability has also an imaginary component, the risetime response is increased limiting the applications on frequency range, particularly the maximum frequency value.

\section{Experimental Material and method}

\subsection{Material}

The device presented in the above sections has been designed and built for recording fast currents fluxing in a plane line. It is made by a $\mathrm{Cu}$ wire wound along a Lucide rod of $4 \mathrm{~mm}$ diameter $27 \mathrm{~mm}$ long. The wire has a $0.11 \mathrm{~mm}$ in diameter which provides a maximum value of turn density of $80 \mathrm{rings} / \mathrm{cm}$ with a total of 216 turns. The coil load $R_{C}$ was the characteristic impedance of a 50 $\Omega$ cable, while the value of $R$ has been measured to be of $4 \Omega$.

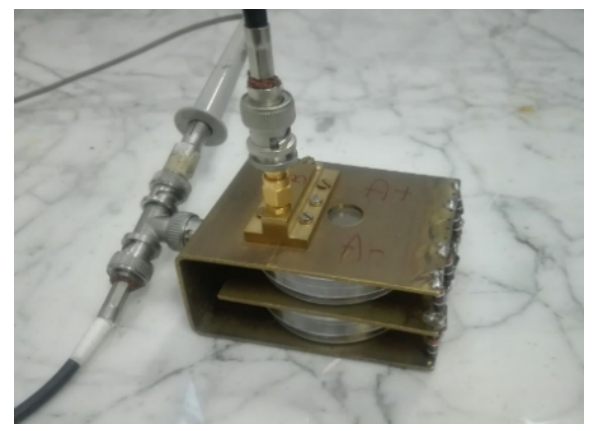

Fig. 11: Double plane transmission line with the linear Rogowski probe on the ground conductor. The transverse dimension is $6 \mathrm{~cm}$.

The transmission line we used in this work was a double transmission line of $100 \Omega$ characteristic impedance connected in parallel as can be seen in Fig. 11. Both the lines are composed by two plane $\mathrm{Cu}$ conductors $6 \mathrm{~cm}$ wide, spaced $2 \mathrm{~cm}$. The end of each line is closed on a resistive load by means of 4 parallel resistors of $400 \Omega$, equal distributed. Similar configurations are present in the laser system MOPA and in biophysical applications.

Under this configuration, the current in each line is the half of the total current and Eq. 22 is modified in

$$
v_{R c}(t) \cong \frac{I_{0} R_{c}}{2 n} \frac{d}{a} e^{-\frac{R}{L} t} \cdot u(t)
$$

In practice the expression (23) might contain the value of $R_{c}$ and, being the value of $L$ of the order of $500 \mathrm{nH}$, the time duration of the signals we can record may be of at max $200 \mathrm{~ns}$.

The Rogowski coil is applied on the ground conductor of the plane transmission line. The slit operated in correspondence of the coil was $35 \mathrm{~mm}$ long in order to guide the input current around the coil.

\subsection{Results}

The behaviour of the probe was studied by means of a current pulser made by a high voltage power supply connected to a coaxial cable (triax cable Suhner $50 \Omega$ ) and a fast switch (Reed relay h12). The output of the pulser was applied to the double transmission line and the provided current was monitored by means a high impedance resistive probe having an attenuation of 46 , see Fig. 12. Instead, the Rogowski coil output was closed to a $50 \Omega$ coaxial cable utilised also to connect the output to oscilloscope, $2 \mathrm{GHz}$ (20GS/s). 


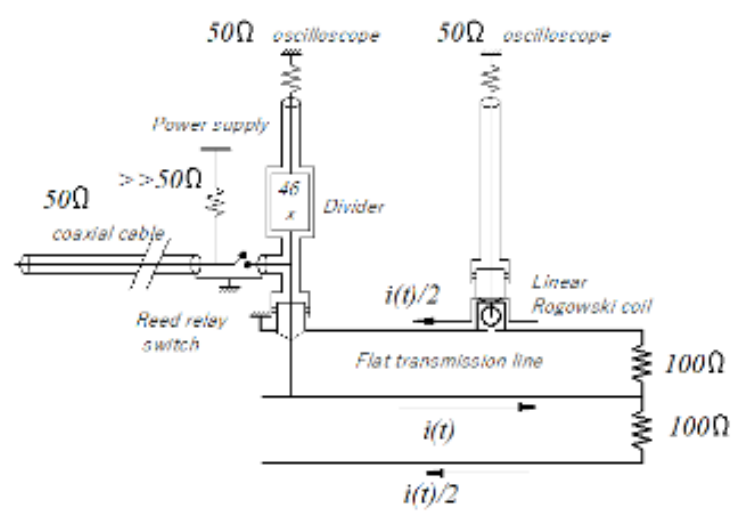

Fig. 12: Sketch of the electric circuit used to calibrate the linear probe.

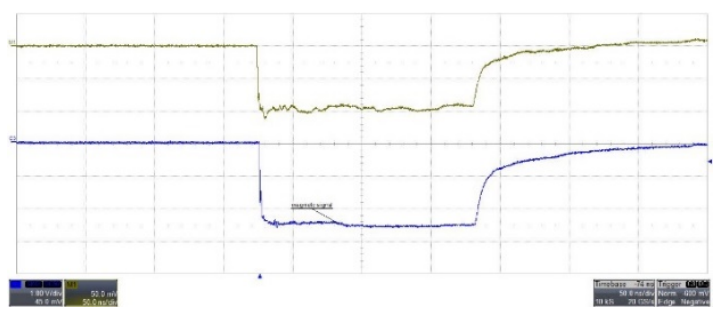

Fig. 13: Waveform of the input and output signals. Upper lower is the input current; upper trace is the output signal. Time scale $50 \mathrm{~ns} / \mathrm{div}$.

The waveform response is very near to the input one, as can be seen in Fig 13, where we have used one pulse forming line of $16 \mathrm{~m}$.

The lower trace scale is $1 \mathrm{~V} / \mathrm{div}$ which correspond to $1 \mathrm{~V} \times \frac{46}{50 \Omega} \frac{1}{2}=0.46 \mathrm{~A} / \mathrm{div}$, while the upper trace is 50 $\mathrm{mV} / \mathrm{div}$ while correspond to $50 \mathrm{mV} / \mathrm{div}$. The attenuation factor is about $12 \mathrm{~A} / \mathrm{V}$, while theoretically one expect $216 \times \frac{60}{27} \times \frac{1}{50}=9.6 \mathrm{~A} / \mathrm{V}$.

The discrepancy we found is mainly due to the distributed current inside the line owing to the slit that we have operate only for $50 \mathrm{~mm}$. The maximum time duration of the detection signal is about $157 \mathrm{~ns}$, as can be seen in Fig. 13.

In order to test the behaviour of our device, we perform an experiment with very short pulse utilising as pulse forming line of about $5 \mathrm{~cm}$. The result of the latter test are illustrated in Fig. 14, where the time scale is $1 \mathrm{~ns} / \mathrm{div}$.

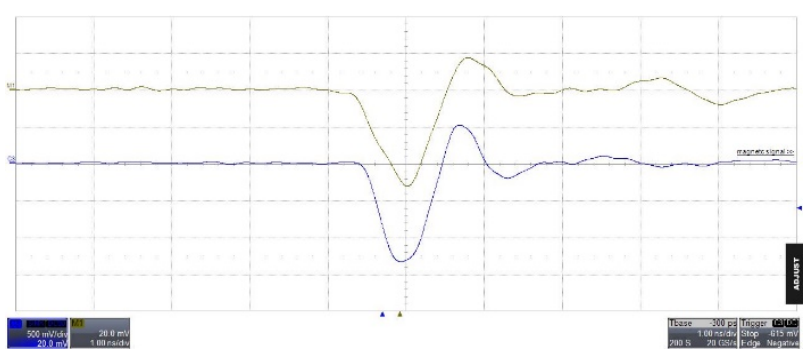

Fig. 14: Experimental result: Upper trace is the input signal of 650 ps FWHM $(0.5 \mathrm{~V} / \mathrm{div})$; Lower trace is the output signal of $700 \mathrm{ps}(20 \mathrm{mV} / \mathrm{div})$. Time scale $1 \mathrm{~ns} / \mathrm{div}$.

If we turn our attention to the fast rise time of the response, we find very interesting results, represented in Fig. 15, with a $500 \mathrm{ps} / \mathrm{div}$ time scale. From Fig. 15, the input signal presents a rise time of about $500 \mathrm{ps}$, while the output signal of about $600 \mathrm{ps}$. Therefore, the discrepancy is of about $100 \mathrm{ps}$, that we can ascribe to response quality of the Rogowski coil.

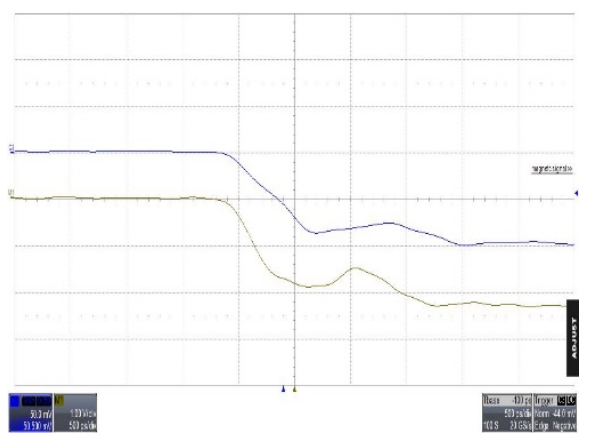

Fig. 15: Experimental result: Upper trace is the input signal (1 $\mathrm{V} /$ div); Lower trace is the output signal $(50 \mathrm{mV} / \mathrm{div})$. Time scale $500 \mathrm{ps} / \mathrm{div}$.

If we turn our attention to the fast rise time of the response, we find very interesting results, represented in Fig. 15, with a 500 ps/div time scale. From Fig. 15, the input signal present a rise time of about $500 \mathrm{ps,} \mathrm{while} \mathrm{the}$ output signal of about $600 \mathrm{ps}$. Therefore, the discrepancy is of about $100 \mathrm{ps}$, that we can ascribe to response quality of the Rogowski coil.

\section{Conclusion}

By this device it is possible to diagnose fast current pulses in plane conductors. The response quality might be improved using ferromagnetic cores. The attenuation coefficient is independent by the magnetic permittivity and the disagreement we found between the expected and measured attenuation factor is mainly ascribed to the length of the slit we operated on the line conductor. The calculations developed above show how the wire resistance and the load resistance do not affect the rise time.

\section{References}

1. W. Rogowski and W. Steinbhaus, Arch. Electrotech. 1, 141-50 (1912) 
2. A. Luches, V. Nassisi and M.R. Perrone, J. Phys. E: Sci. Intrum. 20, 1015-1018 (1987)

3. F. Belloni, V. Nassisi, P. Alifano, C. Monaco, A. Talà, M. Tredici and A. Rainò, Rev. Sci. Instrum. 76, 0543021-6 (2005)

4. D. A. Ward and J. L. T. Exon, Eng. Sci. Edu 2, 10513 (1993)

5. J. Cooper, Plasma Physics. 5, 285-89 (1963)

6. V. Nassisi and A.Luches, Rev. Sci. Instrum. 50, 90002 (1979)

7. R.Y.Han, W.D. Ding, J.W. Wu, H.B. Zhou, Y. Jing, Q.J. Liu, Y.C. Chao, A.C. Qui, Rev. Sci. Instrum. 86, 035114 (2015)

8. R.H. Uddlestone, S.L. Leonard, Plasma diagnostic technique, Academic Press, New York (1965)

9. D. G. Pellinen, Rev. Sci. Instrum. 42, 667-9 (1971)

10. V. Nassisi, P. Alifano, A. Talà and L. Velardi, J. Appl. Phys. 112, 014702 (2012)

11. D. Delle Side, L. Velardi, V. Nassisi, Appl. Surf. Sci. 272, 124-127 (2013)

12. S. Agosteo, M.P. Anania, M. Caresana et al., Nucl. Instr. Meth. Phys. Res. B 331, 15-19 (2014).

13. J.M. Anderson, Rev. Sci. Instrum. 42, 915-30 (1971) 